\title{
Gläubige Zweifler. Eine Fallanalyse zur medialen Inszenierung freikirchlicher Religiosität
}

\author{
Adrian Totaro
}

Eingegangen: 7. Januar 2021 / Überarbeitet: 5. August 2021 / Angenommen: 16. August 2021 / Online publiziert: 4. Oktober 2021

(C) Der/die Autor(en) 2021

Zusammenfassung Religion wandelt in sich modernisierenden Gesellschaften ihre Form und wird zur Wahl; Gemeinden suchen in medialen Arrangements zunehmend nach Aufmerksamkeit. Im Zuge der Pluralisierung stehen sie vor zwei zentralen Herausforderungen: einerseits der Auseinandersetzung mit einer säkularen Gesellschaft und andererseits der Konkurrenz mit anderen Sinnanbietern. Der Beitrag diskutiert anhand eines exemplarischen Falls eine spezifisch kommunikative Problembearbeitung im religiösen Feld. Mit der Wissenssoziologischen Videohermeneutik wird das Webvideo einer christlich-freikirchlichen Gemeinde auf seine mediale Gestalt, das räumliche Setting und die darin körperlich und sprachlich vollzogene Handlung hin untersucht. Die Analyse rekonstruiert auf der medialen Ebene ein Wechselspiel zwischen vertrauten Formen massenmedialer Kommunikation und der Kreation von etwas Eigenem sowie inhaltlich die Auseinandersetzung zwischen Religion und Säkularität. Abschließend werden das dem Datum inhärente Inszenierungsmoment und der über den Fall hinausverweisende Inszenierungstypus eingeordnet.

Schlüsselwörter Soziologie · Religion · Pluralismus · Wissenssoziologische Videohermeneutik $\cdot$ Mediale Inszenierung $\cdot$ Freikirche

\footnotetext{
Adrian Totaro $(\bowtie)$

Institut für Sozialwissenschaften - Abteilung Soziologie, Universität Koblenz-Landau, Landau in der Pfalz, Rheinland-Pfalz, Deutschland

E-Mail: totaro@uni-landau.de
} 


\section{Devout doubters. An analysis refering to medial staging of free church religiousness}

Abstract In modernizing societies religion changes its form. It becomes a choice. Religious communities are looking for attention by using different types of media. However, as a result of pluralism we are confronted with two main challenges: on the one hand, we have the confrontation with the secular sphere and, on the other hand, the competition between different religious providers. The article discusses the topic of handling specific problems of communication by examining an individual case. Using the Wissenssoziologische Videohermeneutik, a Webvideo of a Free Church in Germany is evaluated in its form of media, its spatial setting and the presentation of physical and linguistic expressions. The evaluation of the material shows an interplay in media between familiar forms of mass media and the creation of something own. And in regard to the context, the examination is performed by the interplay of secularity and religion. Finally, the article ends with the discussion on the moment of staging and type of staging.

Keywords Sociology $\cdot$ Religion $\cdot$ Pluralism $\cdot$ Video hermeneutics $\cdot$ Media production $\cdot$ Free church

\section{Einleitung}

Religion hat eine ,wesentliche Funktion zur Konstruktion, Aufrechterhaltung und Veränderung der sozialen Ordnung“ (Knoblauch 2006, S. 277). Sie bietet geordnete Lösungen von Alltagsproblemen ebenso wie Möglichkeiten der Bewältigung kritischer Lebenssituationen. Als sozio-historisches Gebilde ist sie „von Menschen erdacht, in Kommunikation ,offenbart', in Handlungen realisiert und von Experten zu Sinnsystemen ausgebaut" (Knoblauch 2006, S. 278). Ihre herausragende Bedeutung für die Gesellschaft betonen auch Peter Berger und Thomas Luckmann, weshalb sie die Religionssoziologie als ,,integrale[n] und sogar zentrale[n] Bestandteil der Wissenssoziologie“ (Berger und Luckmann 2020, S. 29) sehen und ihre Aufgabe in der „Analyse des kognitiven und normativen Apparats, durch den das sozial konstituierte Universum (genauer: das Wissen über dieses Universum) legitimiert wird“ (ebd.). Eine Religionssoziologie dürfe sich daher nicht auf Kirchen und deren Besucherzahlen beschränken, sondern müsse vielmehr nach der gesellschaftlichen Bedeutung der Religion für die Legitimierung gesellschaftlicher Universa fragen.

Herrschten lange Zeit Prognosen und Theorien des gesellschaftlichen Niedergangs der Religion vor, so ist zu sehen, dass - wie Thomas Luckmann bereits in den 1960er-Jahren in der Unsichtbaren Religion (2014) festhält - sie keineswegs verschwindet, sondern vielmehr ihre Form wandelt. Auch Peter Berger griff in seiner Schrift Zwang zur Häresie (1980) jene Entwicklungen auf, die unter den Stichpunkten Globalisierung, Individualisierung und Pluralisierung Einfluss auf das gesellschaftliche Leben und die Religion haben. Schließlich werde die Religion zur Option, die Mitglieder werden nicht mehr zwangsläufig in Religionsgemeinschaf- 
ten hineingeboren, sondern können unter dem Aspekt der Wahl hinzutreten oder sie ablehnen.

Nachdem Berger jedoch auch lange Zeit die These der Säkularisierung vertreten hatte, revidierte er sie in der These der Zwei Pluralismen (vgl. Berger 2015; auch 2017): Zwar unterminiere der Pluralismus religiöse Gewissheit und eröffne eine Vielzahl an Wahlmöglichkeiten, Religion verschwinde jedoch nicht. Vielmehr seien im „Großteil der Welt [...] diese Wahlmöglichkeiten [...] auch religiöse“ (Berger 2015, S. 39). Es sei daher nicht nur auffällig, dass mehrere Religionen nebeneinander existierten, sondern dass sie zugleich mit einem säkularen Diskurs koexistierten, das heißt, dass Religion auf der einen Seite Religion(en) gegenübersteht und auf der anderen Seite mit Säkularität konfrontiert ist. Die eigentlich Gewissheit versprechende Religion und ihre Legitimationen werden zum Problem, weil auch der Glaube zum Gegenstand der Reflexion, einer Entscheidung, wird und ,immer von einem Halbschatten des Zweifels umgeben“ sei (Berger 2017, S. 19), eine Herausforderung, der sich sowohl Individuen als auch Institutionen stellen müssen (vgl. Berger 2017, S. 20). Lassen sich einerseits Erfahrungen von Transzendenz vielfach auch außerhalb der Religion bewältigen, was Hubert Knoblauch unter anderem in der Ekstatischen Kultur beschreibt (Knoblauch 2001), verweisen andererseits charismatische Bewegungen, als die weltweit am stärksten wachsenden Strömungen im Christentum (vgl. Zimmerling 2018), auf die weite Verbreitung religiöser Antworten.

Daneben haben Prozesse der Medialisierung und Digitalisierung Auswirkungen auf die Sozialform der Religion. ${ }^{1}$ Ist die Frage nach der Religion mit Fragen der Bedeutung von heiligen Orten und Räumen, Materialitäten, Handlungs- und Interaktionspraktiken verbunden, ist in diesem Zusammenhang zu fragen, ,ob Partizipation an religiösen Gemeinschaften, die zumeist als körperliche Kopräsenz der Gläubigen konzipiert wird, nicht in neue mediatisierte Formen verwandelt werden kann“, wie Knoblauch in seinem Aufsatz zur Kommunikativen Konstruktion der Transzendenz (2017, S. 238) vermutet. Bei dem hier zu untersuchenden Fall ist die Frage zunächst darauf zu beschränken, wie religiöse Gemeinschaften medial nach Aufmerksamkeit suchen, welche Inszenierungsweisen sie verwenden und welche Bedeutung diese Darstellungs- und Inszenierungsformen tragen. ${ }^{2}$

Der Bibel-Clip oder das Wort zum Sonntag sind dabei nur zwei aus der massenmedialen Kommunikation bekannte und vertraute Formen kirchlicher Verkündigung. Reichertz gibt in Bezug auf den Bibel-Clip jedoch zu bedenken, dass dieser die Religion in einen Zusammenhang mit Werbung rücke, weil Religion zu einer Ware und Gott damit wählbar oder abwählbar werde, wenn jene kulturelle Technik der Verbreitung und Anwerbung zur Aufmerksamkeitssteigerung eingesetzt wird (vgl. Reichertz 2002). Die zunehmende Nutzung digital-medialer Formen zur Verbreitung

\footnotetext{
${ }^{1}$ Unter Medialisierung sei ein Prozess der Veränderung kommunikativer Praktiken verstanden: „,Vergesellschaftungsbereiche verändern sich intern durch Massenmedien (z. B. Politik, Erziehung und Familie); und Vergesellschaftungsprozesse verändern sich für Massenmedien (z. B. Wissenschaft, Sport, Politik und ansatzweise auch die Kirchen)“ (Ziemann 2018, S. 66). In Hinsicht auf Digitalisierung sei an dieser Stelle auf die zunehmende Bedeutung digitaler Technik- und Kommunikationsformen innerhalb der Gesellschaft verwiesen (vgl. Weyer 2017).

2 Zum Einfluss technischer Veränderungen im Alltag in Bezug auf die Religionsausübung vgl. Herbrik (2013).
} 
von Botschaften und die relativ einfache Handhabung der Produktion und Verbreitung, beispielsweise über video-sharing-Websites, ermöglichen religiösen Akteuren, ihre Botschaften an ein breites Publikum zu verbreiten. ${ }^{3}$

Dieser Beitrag untersucht den Aspekt medialer Inszenierungen religiöser Gemeinschaften anhand eines exemplarischen Falls mithilfe der Wissenssoziologischen Videohermeneutik. Ziel ist die Rekonstruktion jenes Falles und die Entwicklung einer Strukturhypothese über die Probleme, Herausforderungen und Lösungen kommunikativen Handelns. Die Strukturhypothese beschreibt das Allgemeine im Besonderen und bildet die Grundlage für eine idealtypische Fallbeschreibung im Sinne Max Webers und Alfred Schütz'. ${ }^{4}$ Drei Fragen leiten die Analyse an: Wie ist das mediale Produkt aufgebaut, und an welchen kommunikativen Formen ist es orientiert? Welche Bedeutung tragen sein räumliches Setting und der darin dargestellte körperliche Ausdruck in Verbindung mit den verbalen Äußerungen des Protagonisten? Und welches Inszenierungsmoment liegt im Zusammenwirken der drei Ebenen vor? Nach der Erläuterung des methodischen Vorgehens (2) wird der empirische Fall beschrieben, interpretiert und eingeordnet (3) und abschließend ein kurzer über den Fall hinausweisender Ausblick gewagt (4).

\section{Wissenssoziologische Videohermeneutik}

Grundlegend für eine methodisch kontrollierte Analyse empirischer Daten ist die Wahl der angemessenen Methode für das Material. Audio-visuelle Daten stellen wegen ihrer komplexen Überschneidungen von Simultaneität - der Gleichzeitigkeit im Bild - und Sequenzialität - dem temporalen Voranschreiten - der Zeichen und Symbolformen für die sozialwissenschaftliche Analyse eine Herausforderung dar, denn „[a]nders als für die Deutungslehre von Schrifttexten, die auf eine jahrhundertelange Tradition zurückblicken kann, bestehen für die verhältnismäßig junge Disziplin der Hermeneutischen Film- und Videoanalyse nach wie vor eine ganze Reihe ungelöster Grundprobleme“ (Raab 2008, S. 156). Schließlich weist das Material Handlungen vor der Kamera, Handlungen der Kamera sowie Techniken der Nachbearbeitung auf, die ineinanderfließen (vgl. ebd.). Aufbauend auf Thomas Luckmann und HansGeorg Soeffner überführt Raab die Überlegungen einer hermeneutisch orientierten Wissenssoziologie, die auf dem ,bewusste[n] und methodisch kontrollierte[n] Einsatz von Techniken und Methoden einer von Menschen immer schon praktizierten ,Alltagshermeneutik“" basiert, auf die Analyse von Videomaterial (Raab 2008, S. 135). ${ }^{5}$

Ausgehend vom interpretativen Paradigma einer methodisch kontrollierten Rekonstruktion gesellschaftlicher Wirklichkeit und deren Interpretation (vgl. Raab und Stanisavljevic 2018, S. 58) baut die Wissenssoziologische Videohermeneutik auf die sich bereits im Alltag als normal und selbstverständlich bewährten Verstehensarbeit

\footnotetext{
3 Dazu zählen auch eigene Homepages, Social Media Accounts etc.

4 Vgl. Oevermann (2000), Soeffner (2004), Wernet (2019).

5 Zur Anbindung der wissenschaftlichen Einstellung in Bezug auf die Alltagsauslegung vgl. Soeffner (2004).
} 
auf, um Aufschluss ,über die historisch, kulturell und sozial geprägten Selbst- und Weltdeutungen von Gruppen, Gemeinschaften und Gesellschaften“ zu geben (ebd.). Die Frage richtet sich auf die Konstruktion sozialen Sinns und sozialer Bedeutung und kann sowohl Handlungen als auch Texte, Bilder und Videos einschließen. Schließlich gründen kommunikatives Handeln und soziale Beziehungen ,auf der intersubjektiven Aushandlung und Absicherung von sozialem Sinn und sozialer Bedeutung" (Raab und Stanisavljevic 2018, S. 59). Dem im Alltag oftmals abgekürzten Verstehen soll hier gerade eine fixierte, kontrollierte und extensive Interpretation und Deutung entgegengesetzt werden.

Da jedes Dokument seine je eigenen Spuren trägt, ist das Ziel, das „spezifische Sinnpotential eines dokumentierten kommunikativen Darstellungs- und Ausdruckshandelns“ herauszuarbeiten (Raab und Stanisavljevic 2018, S. 61). Die Gleichzeitigkeit im Bild, oftmals verbunden mit symbolisch-schriftlichen Einblendungen und einem darüber gelegten Ton, stellen eine Herausforderung dar, die im Zugleich von Temporalität (Zeitlichkeit) und Simultaneität (Gleichzeitigkeit) unterschiedlicher Handlungsebenen und Symbolformen liege. Das Vorgehen verlangt einen kontextfreien Einstieg, der zugleich die Sequenzialität berücksichtigt und angelehnt an Anselm Strauss die Analyse ,line-by-line“ erlaubt (Raab und Stanisavljevic 2018, S. 63) sowie in der Kontrastierung gesättigt wird. Videodaten werden hierzu zunächst in einer tabellarischen Form als Partitur erfasst, um einzelne „Handlungsund Darstellungselemente des audiovisuellen Datums nach Maßgabe ihrer Problemund Fragestellung sowie gemäß der spezifischen Erscheinungsgestalt des Datums“ zu ordnen (Raab und Stanisavljevic 2018, S. 66).

Die Analyse folgt der Sequenzialität des Datums, das zunächst kontextfrei interpretiert und kontrastiv auf andere Phänomene bezogen wird; es werden hypothetische Kontexte erstellt und mögliche Bezüge hergestellt, wohin der Einzelfall aus sich heraus verweist. Die gebildeten Lesarten werden so sukzessive gebildet und überprüft, um sie zu verfeinern oder auszuschließen. Unter Hinzuziehung von Kontextwissen geschieht hiernach die Erweiterung zur „Strukturhypothese“ (Raab 2008, S. 163), woran die Idealtypenbildung anschließt, die die Strukturelemente zu einem Typus zusammenführt. ${ }^{6}$

Es geht an dieser Stelle nicht darum, was ein religiöses Video auszeichnet, sondern zunächst um die Erfassung inhärenter Merkmale und ihrer Deutungspotentiale des einen Falls. Das Video stellt dabei die Antwort auf ein kommunikatives Problem dar, das gelöst wird und für eine spezifische kommunikative Problembearbeitung im religiösen Feld (Bourdieu 2000) steht, die sich als mediale Inszenierung von Religiosität offenbart. Es steht hier exemplarisch und alleine. Die aufgeführte Rekonstruktion kann jedoch im Sinne der Grounded Theory (vgl. Glaser und Strauss 1998; Strauss 2004; auch Strübing 2008) weitere Analysen anleiten.

\footnotetext{
${ }^{6}$ Die folgende Analyse präsentiert dementsprechend verkürzt und pointiert bestimmte Lesarten. Da es sich um die Explikation eines Einzelfalls handelt und ein kontrastierender Fallvergleich an dieser Stelle nicht geleistet werden kann, schließt die Interpretationsarbeit mit einer Zusammenfassung der wesentlichen Strukturelemente der Kommunikation. Im Sinne der Grounded Theory endet der Text mit einer materialen Theorie, der durch Heranziehung weiterer vergleichender Analysen das Potenzial einer formalen Theorie erhält (vgl. Glaser und Strauss 1998, S. 120f.).
} 


\section{Exemplarischer Fall: Das Webvideo einer freikirchlichen Gemeinde}

Das vorliegende Datenmaterial stammt von der Homepage einer freikirchlichen Gemeinde in Deutschland. Sie bindet das Video neben Texten und Bildern in ihre Außendarstellung ein und rahmt es als Einladung. Für das Webvideo ${ }^{7}$ nutzt sie die digitale Infrastruktur einer video-sharing-Website, das Material ist jedoch lediglich über die eigene Homepage zugänglich. Stellen Bibel-Clip und Wort zum Sonntag Beispiele etablierter und professionell erarbeiteter massenmedialer Produkte dar, mit denen vornehmlich die Amtskirchen präsent sind, lässt die digitale Kommunikation eine freiere und individuell gestaltbare Kommunikation zu. Das Betrachten von Internetvideos - aber auch anderen medialen Formen - gehört mittlerweile zum Alltag und verbreitet sich aufgrund niedrigerer Kosten bei Produktion und Distribution weiter (vgl. Traue und Schünzel 2019, S. 1067). Im Folgenden richtet sich der Blick ausschließlich auf das Webvideo. Auf zwei analytischen Ebenen werden der Bildausschnitt: Medium und Raum (3.1.) und die kommunikative Handlung: Körper und Sprache (3.2.) unterschieden. Die Strukturelemente sind schließlich in dem sich zeigenden Inszenierungsmoment zu verdichten (3.3.) und abschließend nach einem allgemeinen Inszenierungstyp im Besonderen zu hinterfragen (3.4.).

\subsection{Medium und Raum}

Der Einstieg in das Material erfolgt über das mediale Arrangement und das räumliche Setting. Für die Ausarbeitung ist es anonymisiert, der Protagonist sowie das eingefügte Gemeindesymbol sind unkenntlich gemacht. Jene Elemente sind für die hier skizzierten Überlegungen zu vernachlässigen, die Analyse konzentriert sich auf die Handlung vor der Kamera.

Das Video hat eine Dauer von zwei Minuten. Es zeigt einen die gesamte Dauer unveränderten Bildausschnitt, in dem ein Mann mit legerer Alltagskleidung, hochgekrempelten Hemdsärmeln, geöffneten Kragenknöpfen, ausgestattet mit einem Lavalier-Mikrofon vor einem von Häuserfassaden unterschiedlicher Baustile eingerahmten Marktplatz steht und in die Kamera spricht (siehe Abb. 1). Der Hintergrund ist verschwommen, der Sprecher scharf gestellt. Die Bildkomposition verweist auf eine spezifische Ordnung: der Protagonist im Mittelpunkt und damit im Fokus, die religiöse Markierung am Rand und die lokale Einbettung verschwommen im Hintergrund. Die Handlung findet ausschließlich vor der Kamera statt, denn diese ist fest arretiert und verharrt auf dem Bildausschnitt. Nach Minute [1:56] verdunkelt sich das Bild und das Gemeindesymbol zieht sich vergrößernd in den Mittelpunkt, wo es zum Abschluss vor weißen wehenden Pixeln aufleuchtet.

Die religiöse Markierung im Bild, der Ausklang durch dessen Vergrößerung und Hervorhebung sowie der durchgängig klare und verständliche Ton deuten auf eine technische Nachbearbeitung hin. Sie unterstreichen im Unterschied zu spontanen Aufnahmen und deren Veröffentlichung eine Planung und Organisation. Auch die Hintergrundgeräusche - das geschäftige Markttreiben oder vereinzeltes Vogelgezwitscher - sind wahrzunehmen, aber keinesfalls störend überlagernd, sondern

\footnotetext{
7 Zur kurzen Übersicht vgl. Traue und Schünzel (2019).
} 


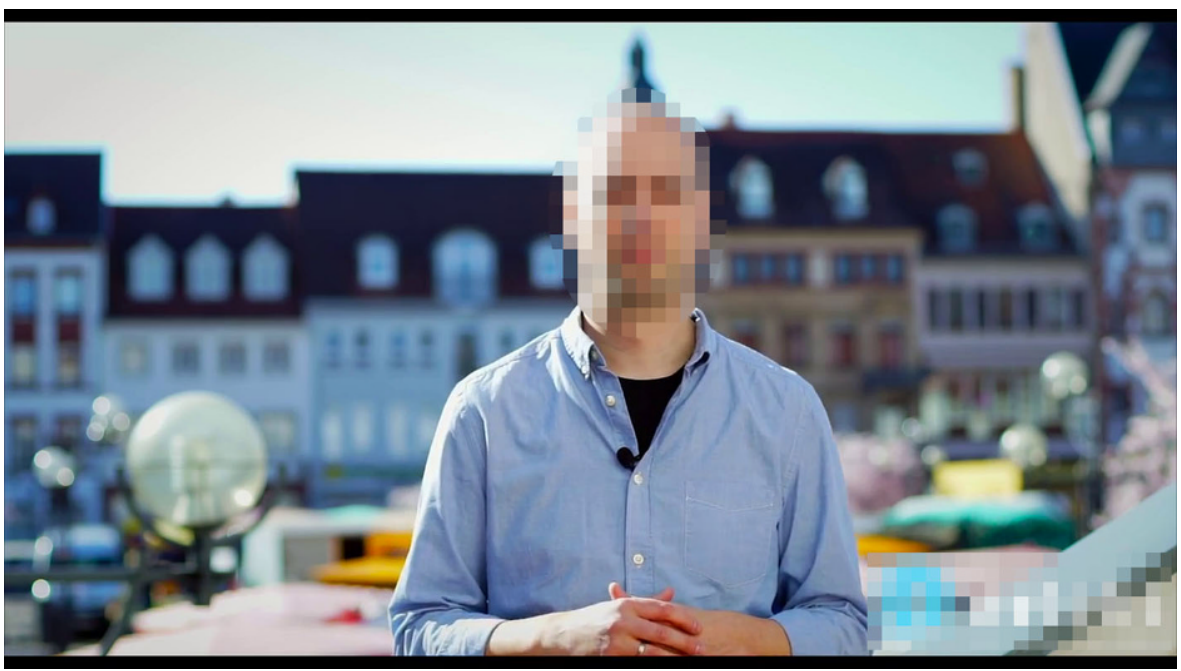

Abb. 1 Video-still [0:03] - Der Protagonist in der aufrechten Grundhaltung im Zentrum des Bildes

vielmehr begleitend. Die unveränderte Kameraperspektive und einfache Gestaltung sind darüber hinaus Anzeichen eines minimalistischen Gebrauchs der eigentlichen medialen Möglichkeiten. Es finden weder Perspektivenwechsel noch Einblendungen statt. Das Video startet und endet mit der gleichen Position, Einstellung und Perspektive.

Entgegen den mittlerweile vielfach genutzten Selfie-Perspektiven, die mit Verwackeln oder Herumreißen der Kamera einhergehen, zeichnet sich die Aufnahme durch ihre Ruhe in der Protokollierung einer Szene aus. Der sequentielle Ablauf ist orientiert an einer spezifischen Ordnung. Die Inszenierung erscheint durchdacht und nicht spontan, die arretierte Kamera und der Bildausschnitt ausgewählt, die ruhige Darstellung trägt Elemente von Seriosität, die beispielsweise mit Reportern oder Kommentatoren am Ort des Geschehens vergleichbar sind.

Die Reduktion und Schlichtheit in der Verwendung medialer Möglichkeiten rückt den Protagonisten ins Zentrum der Aufmerksamkeit und vermittelt seine Botschaft. Die halbnahe Perspektive lässt das Sehen seiner Körperbewegungen zu, reduziert die Sicht aber auf Oberkörper, Arme und Hände. Das Publikum sieht den Ausschnitt eines Sprechers, wie er sich zentral im Bild ausrichtet, bewegt und die rahmende Kulisse des Markplatzes als Bühne gebraucht.

Begrenzt von Häuserfassaden bindet der Platz den Protagonisten lokal ein, ohne eindeutig zu identifizieren, wo er sich befindet. Er steht vor der Balustrade einer nach rechts oben verlaufenden Treppe und steht damit seinerseits oberhalb des Platzes. Er ist damit nicht nur visuell durch Schärfe/Unschärfe von ihm getrennt, sondern zugleich im Bild von ihm erhoben. Er ist nicht Teil der aufgestellten Stände, sondern steht abgetrennt am Rand.

Die Marktstände stehen für geschäftiges Treiben. Zugleich symbolisieren sie einen zentralen Platz einer Stadt oder Gemeinde. Das Bild markiert damit einen Rahmen städtischer Öffentlichkeit. In diesem jedoch wird - das verrät das religiöse 
Symbol - eine religiöse Kommunikation aufgeführt. Der Bildausschnitt zeichnet sich daher in zweifacher Hinsicht aus: Die Kulisse wird einerseits zum Symbol einzigartiger Lokalisation, die einem vertrauten Publikum als spezifischer Ort vertraut ist, für den Ortsfremden aber überall sein kann; andererseits bietet er allgemein ein Symbol der weltlich-säkularen Sphäre. Die im Bild eingefügte religiöse Markierung steht dieser entgegen, als suchte Religion die Welt als Ort und Rahmung ihrer Botschaft. Es findet demnach die Verortung der Religion im öffentlichen Raum statt, die zugleich als Spannungsfeld gedeutet werden kann: Religion im öffentlichen Raum auf der einen Seite und auf der anderen Seite der öffentliche Raum als Symbol der weltlich-säkularen Sphäre und einzigartiger Lokalisation. Visuell zeichnet sich die gleichzeitige Darstellung von lokaler Einzigartigkeit und allgemeiner Öffentlichkeit aus; der eigentlich religionsferne Raum wird zum Ausgangspunkt einer religiös markierten Kommunikation.

Suggeriert das mediale Arrangement einen von Schlichtheit, Planung und Seriosität geprägten Charakter, verortet das räumliche Setting die Situation lokal in der Welt. Visuell werden lokale Einzigartigkeit und weltlich-säkulare Sphäre als Rahmung verwendet, auf die im Hintergrund unscharf verwiesen wird, ohne die jedoch die notwendige Kulisse der Inszenierung fehlen würde. Erhöht über den alltäglichen Dingen der Welt kann der Sprecher ausbreiten, was es zu verkünden gilt. Er positioniert sich gleichsam auf einem Markt, der die Wahl von Möglichkeiten offeriert.

In einem ersten Schritt lässt sich daher festhalten, dass Vorder- und Hintergrund einerseits klar getrennt, andererseits aber ebenso durchlässig erscheinen. Säkulare Welt und religiöse Botschaft treten gemeinsam auf. Die Wahl der Ausdrucksmittel unterstützt bis zu diesem Punkt die These einer auf Seriosität zielenden, Schlichtheit und Einfachheit gebrauchenden und an festen Orientierungsmustern ausgerichteten Inszenierung. Diese gilt es nun, in ihrem körperlichen und sprachlichen Ausdruck zu untersuchen.

\subsection{Körper und Sprache}

Die Kommunikation lässt sich in fünf Sprechakte gliedern, die im Folgenden sequentiell zu explizieren sind. Dem ersten Sprechakt geht eine non-verbale Ausrichtung des Protagonisten im Bildausschnitt voraus. Wie oben angemerkt, schließt das Video mit der Verdunkelung und gleichzeitigen Zentrierung des Gemeindesymbols. Es zeigen sich damit eine körperliche Vorbereitung [0:00-0:01], fünf Sprechakte [0:01-1:55] und die anschließende Sequenz der Zentrierung der organisationalreligiösen Markierung [1:56-2:00]. ${ }^{8}$

Das Video startet mit einer Aufblende, das Bild öffnet sich. Der Protagonist erscheint im Bildzentrum, richtet sich auf, nimmt eine angespannt-aufrechte Haltung ein und atmet hierbei tief ein. Er streckt sich, hebt seine Hände vor den Brustkorb und öffnet die Handflächen leicht nach oben. Er breitet sie vor sich aus. Der Einstieg erfolgt non-verbal als körperliche Ausrichtung im Bild. Einatmen, Haltung einneh-

\footnotetext{
${ }^{8}$ Der Zusammenhang von sprachlichem und körperlichem Ausdruck kann an dieser Stelle nur verkürzt dargelegt werden. Gleiches gilt für die Interpretation der Sprechakte.
} 
men, Arme anwinkeln und Hände ausbreiten stellen eine sichtbare Vorbereitung dar, die vor den Augen des Publikums geschieht. Es sieht, was mit Beginn der Aufzeichnung noch nicht fertig war: die Positionierung des Sprechers. Die aufrechte, zentrale Position wird dabei zu einer die Kommunikation einleitenden Handlung und zugleich als mögliches Ideal der folgenden Bewegungen. Diese deutet auf eine Wichtigkeit hin, die der Protagonist einbringt. Sie verweist zudem auf eine Seriosität, ähnlich der gesamten Gestaltung des Materials. Demgegenüber kann die Eingangssequenz jedoch als erster Bruch gedeutet werden, wenn die körperliche Ausrichtung und Vorbereitung sichtbar wird. Im Vergleich mit professionellen Produkten wie einem Tagesschaubericht ist das eher unüblich. Die Kamera protokolliert jedoch genau das mit.

Betrachten wir nach der körperlichen Vorbereitung den sprachlichen Ausdruck und setzen ihn in Relation zum gleichzeitig vollzogenen körperlichen Ausdruck:

Zweifel? Gehör-n zum Leben dazu. ${ }^{9}$

Der erste Sprechakt wird vom Ausbreiten der Hände betont unterstützt, als präsentieren sie das Gesprochene zusätzlich und heben es hervor. Das trifft insbesondere auf den ersten Ausdruck ,Zweifel“ zu. Der prosodisch zwischen Ausruf und Frage hervorgehobene Begriff wird zum Ausgangspunkt eines unüblichen Interaktionsbeginns. Keine Begrüßung, keine Einleitung, sondern ein zunächst zusammenhanglos gewählter Begriff beginnt den Sprechakt. Handelt es sich um einen Namen, ein Unternehmen, oder meint es die Erfahrung von Brüchigkeit, Unentschiedenheit oder Ungewissheit? Ist es ein Aufruf zu einer bestimmten Geisteshaltung, zu hinterfragen, zu bezweifeln? Sprachlich ist der Beginn höchst ungewöhnlich und könnte innerhalb einer wissenschaftlichen Diskussion ebenso auftauchen wie in der Ausrufung eines Namens. Erst die Fortsetzung verrät, dass es sich nicht um den, sondern um die Zweifel handelt, dass der Begriff eine Brüchigkeit oder Unentschiedenheit bezeichnet, weil sie nun zu einem Attribut des Lebens erklärt werden. Kann der Namenskontext nun ausgeschlossen werden, bleibt die Möglichkeit der wissenschaftlichen Diskussion, in der jedoch keine Definition folgt, sondern jene Einordnung als zum Leben dazugehörend.

Der Beginn mit „Zweifel“ hebt diese sprachlich hervor, sodass sie die folgende Kommunikation bestimmen. Zugleich werden sie als Selbstverständlichkeit fast schon trivialisiert. Der Einstieg erfolgt über eine Gegenüberstellung und Verknüpfung von Zweifel und Leben. Dem möglichen Problem wird seine eigene Selbstverständlichkeit hinzugefügt.

Während sprachlich das Problem trivialisiert beziehungsweise zu einem natürlichen und selbstverständlichen Bestandteil des Lebens deklariert wird, zeigt der Körper eine zweite öffnende Bewegung der Hände, die zunächst ausgebreitet, dann im Abschluss unterhalb des Brustkorbs übereinander gelegt werden. Die angespannt eingenommene Haltung wird entspannt. Der Körper unterstützt gestisch durch Öffnung und Schließung gegenüber dem Publikum die Präsentation des Sachverhalts als Problem und Selbstverständlichkeit zugleich. So findet die Bewegung zunächst ihre Ruheposition in der anfangs beschriebenen idealen Haltung. Mit Übergang zum

\footnotetext{
9 Timecode [0:01-0:03].
} 
zweiten Sprechakt wird diese wiederum gebrochen, weil der Oberkörper zur Seite schwingt. In der Sprechpause ${ }^{10}$ findet wie zu Beginn eine Orientierung und Ausrichtung statt. Sprachlich schließt hieran an:

Wenn Menschen ernsthaft anfangen=sich mit dem christlichen Glauben zu beschäftigen=entsteh-n automatisch Fragen, wie (--) woher wissen wir über $\downarrow$ haupt, dass Gott existiert?=der (--) wie kann ein guter Gott so viel Leid und Böses zulassen ${ }^{11}$

Der Sprechakt leitet direkt in eine religiöse Thematik ein und markiert sie als dezidiert christlich. Konditional eingeleitet wird eine Beschäftigung mit dem christlichen Glauben als Ursache anschließender Fragen bezüglich der Vereinbarkeit irdischer Erfahrungen und religiöser Vorstellungen. Explizit werden Fragen nach der Existenz Gottes und dessen Zulassen von Leid und Bösem gestellt, die auf das Problem der Theodizee verweisen. ${ }^{12}$

Zwei Aspekte sind an dieser Stelle bemerkenswert: Zum einen ist es der Automatismus der Generierung von Fragen, der in einer ernsthaften Auseinandersetzung gründet; zum anderen ist es der augenscheinliche Gegensatz zwischen der betont ernsthaften, das heißt gewissenhaften und im Ernst betriebenen - und nicht oberflächlichen - Auseinandersetzung mit dem Glauben und den eher trivial wirkenden Fragen nach der Existenz, wenngleich damit grundlegende Fragen an die Religion zum Ausdruck kommen. Der konditionale Einschub setzt aber eine ernsthafte Auseinandersetzung voraus. Sie ist Bedingung und damit der Einstieg in die Auseinandersetzung mit diesen Fragen.

Wie im ersten Sprechakt zeigt sich damit die Gegenüberstellung und Verknüpfung von Problem und Selbstverständlichkeit. Fragen entstehen automatisch, sind damit einerseits erzwungen, zugleich aber völlig normal, sie sind Folge der Auseinandersetzung mit dem Glauben ${ }^{13}$ und seiner Plausibilität. Löste der erste Sprechakt das Problem durch seine Trivialisierung auf, dreht der zweite Sprechakt diese Sicht um: Die mögliche Lösung - die Religion - verursacht ihrerseits neue Fragen. Entgegen der Selbstverständlichkeit von Zweifeln im Leben sind die automatisch resultierenden Fragen jedoch keine beruhigende Lösung mehr, sondern stellen vielmehr selbst neue Probleme dar, worunter die Auseinandersetzung mit den religiösen Dogmen und den Erfahrungen mit der Welt fallen.

Körperlich wiederholt sich die Ausgangsgestalt: Von der zentralen, aufrechten Position heraus werden die Hände als gestische Mittel der Betonung und Präsen-

\footnotetext{
10 Timecode [0:03-0:04].

11 Timecode [0.04-0:17].

12 Nach Weber behandelt es die rationale Bewältigung des Leidens sowie das Setzen von Sinn (vgl. zu einer idealtypischen Beschreibung $\$ 8$ in Weber 2019). Auch Berger führt das Problem der Theodizee zu einem allgemeinen soziologischen Problem aus, wenn es um die Errichtung des Nomos gegenüber dem Chaos geht und dessen Legitimation (vgl. Berger 1973, S. 52ff.).

13 Dass hier von Glaube und nicht von Religion gesprochen wird, erscheint als deutlicher Hinweis auf eine individuelle Auseinandersetzung und zugleich als mögliche Distanzierung zur sozialen Gestalt der Religion. Von der universell-allgemeinen Erfahrung des Zweifels im Leben, der Auseinandersetzung von Menschen, verweist der Glaube stärker als die Religion auf die Verknüpfung individueller Praxis und kollektiver Dogmen und Vorstellungen in einem alltagssprachlichen Verständnis.
} 
tation des Gesagten eingesetzt. Ihre offene Haltung kann auch als ein Abwägen gesehen werden, das den Zusammenhang von Problem und Selbstverständlichkeit offen hält. Zugleich wirkt der Körper jedoch ruckartiger in seiner Bewegung, er wippt vor und zur Seite. Dennoch halten sich die Abweichungen und Ungenauigkeiten in einen engen Rahmen, pendeln mehr um die Ausgangsposition und kehren zu ihr zurück. Mehrmals gehen die Blicke an der Kamera vorbei und visieren etwas daneben Stehendes an. Anders als ein Nachrichtensprecher oder Reporter verliert der Protagonist den Kontakt zum Publikum, und sein Schwanken verrät eine geringere professionelle Übung. Die sofortige Rückbindung zum Publikum sucht diesen Umstand zu kaschieren. Seine körperlichen Bewegungen deuten daher bereits in einem frühen Stadium Anzeichen von Anstrengung an, die zu Ermüdung oder Erschöpfung führen. Die im ersten Sprechakt noch sehr deutliche Übereinstimmung von sprachlichem und körperlichem Ausdruck geht langsam auseinander.

Nach dem allgemeinen Einstieg greift der zweite Sprechakt ein religiöses Problem auf, wenngleich dieses noch allgemein und unspezifisch gehalten ist. Körperlich zeigen sich erste Anzeichen von Anstrengung und Ermüdung, zugleich aber auch die nach jeder Abweichung stattfindende Rückkehr zur Idealfigur, einer zentralen Ruheposition, aus der heraus der nächste Sprechakt vorbereitet wird. Die erneute Ausrichtung und Orientierung an der zentralen und aufrechten Haltung leitet schließlich den dritten Sprechakt ein:

Viele=denken heute, dass man solche und ähnliche Fragen in einer Kirche nicht stellen darf; in=einer Kirche darf man nicht zweifeln,=denn (-) dort glaubt man ja; in einer Kirche, muss=ich glauben, sonst gehör=ich da nicht hin; und wenn=ich vielleicht schon jahrelang glaube (-) und dann=irgendwelche Fragen auftauchen und Zweifel ent $\uparrow$ steh-n, dann muss ich sie möglichst verstecken. ${ }^{14}$

Der dritte Sprechakt vollzieht eine thematische Konkretisierung und leitet zum eigentlichen Problem über. Ausgehend von einer anonymen Zeugenschaft (,viele“) wird eine Annahme formuliert, die das Problem in der Gegenüberstellung von Fragen und Zweifeln einerseits sowie Kirche und Glauben andererseits sieht. Glaube, Kirche, Religion werden dem Fragenden und Zweifelnden gegenübergestellt, der dort keinen Platz finden kann. Dabei vollzieht sich ein Weg personaler Zuschreibung vom Allgemein-Abstrakten zum Konkret-Persönlichen, indem den Erfahrungen - oder Vermutungen - eines generalisierten Anderen im Text das Ich folgt. Das allgemeine Problem, der Ausschluss von Fragen in der Kirche, Zweifel versus Glauben, wird zu einem persönlichen, weil ich als Nicht-Gläubiger und Fragender nicht dazugehören kann oder mich - bin ich bereits Teil davon - mit diesen Fragen verstecken muss. Sowohl Außenstehende als auch Teilnehmer, Gläubige und NichtGläubige werden angesprochen und mit dem gleichen Problem konfrontiert: der Vereinbarkeit von Fragen und Zweifeln mit Glauben und Kirche. Aus dem allgemeinabstrakten Problem wird auf diesem Weg ein individuell-persönliches.

Ziehen wir die drei Sprechakte zusammen, konkretisiert sich das Kommunikat in einer allgemein-universellen Gegenüberstellung und zugleich einer Verknüpfung von Zweifeln und Leben zu einer möglichen religiösen Antwort, die jedoch unsicher er-

14 Timecode [0:17-0:42]. 
scheint und zudem ihren angestammten Platz, die Kirche als Ort und Gemeinschaft, verloren hat, weil dort diese Fragen und Probleme nicht gestellt werden dürften. Das Problem entfaltet sich von der Welterfahrung über die Glaubenserfahrung hinein in die Kirche. Es findet eine sprachliche Zuspitzung statt, die in einem konkreten Problem mündet: dem Ausschluss von Fragen und Zweifeln in der Kirche.

Bezieht man den körperlichen Ausdruck ein, so wird dieser selbst zum Problem: Der Protagonist schwankt, seine Bewegungen werden eruptiver, die Hände fallen aus dem unteren Bildrahmen heraus, er pendelt zunehmend um die Mittelachse und die Blicke schauen - auch während des Sprechens - immer häufiger und länger an der Kamera vorbei. Und dennoch findet er stets zur Ausgangsgestalt zurück. Die Abweichungen erscheinen daher wesentlich als Variationen der idealen Gestalt, als Folge einer Anstrengung und Ermüdung. Geprägt ist diese Figur von der Ausrichtung und Positionierung, Abweichung und stetiger Rückkehr zum Ideal einer aufrechtangespannten Haltung im Bildzentrum. Es ist diese Figur, die sich bis zum Schluss wiederholt, weshalb nun ausschließlich der Text fokussiert werden kann.

Mit dem vierten Sprechakt bricht die bisherige Schilderung ab:

Wir, teilen diese Meinung nicht. (-) Im=Gegenteil (--) wir möchten uns gerne auseinandersetzen mit den Zweifeln (-) die skeptische=Menschen hier in [Stadt] hab $\downarrow$ en=wir möchten uns auseinandersetzen mit den Fragen, die sich Menschen im=Blick auf den christlichen Glauben heute stellen. Weil wir=denken=dass (-) sowohl zweifelnde=Skeptiker als auch gläubige $=$ Menschen es sich schuldig sind, die Grundlagen ihrer Glaubensüberzeugungen zu prüfen. Warum glaub=ich überhaupt, was ich glaube? Und sind meine Zweifel berechtigt? (--) Wir wollen $\uparrow$ Möglichkeiten bieten für diese Art der ehrlichen, respektvollen Auseinandersetzung über die wirklich wichtigen Fragen des=Lebens. Und deshalb bemüh-n wir uns skeptische Zweifel und kritische Rückfragen $\mathbf{z u}=$ versteh-n, (-) und auch auszuhalten. ${ }^{15}$

Dem zuvor mühsam konstruierten Problem wird die Lösung gegenübergestellt. Der organisationale Charakter wird im personalen Wir verdichtet, der sich gegen die weitverbreitete Meinung stellt. Fragen und Zweifel sind nicht auszuschließen, sondern vielmehr in die Kirche einzubeziehen. Mit der Darlegung der eigenen Überzeugung bricht das Kommunikat in zweifacher Weise: Aus der anfangs abstrakten Gegenüberstellung von Zweifel, Leben und Glauben wird eine Lösung im Hier und Jetzt geliefert, die sich an einem Ort und in einer Gemeinschaft spiegelt. Der zweite Bruch findet in der Begründung statt: Schließlich wird der Grund der Auseinandersetzung im Individuum selbst verortet, das es sich schuldig sei, seine Überzeugungen - welcher Art auch immer - zu prüfen. Wird mit der Gegenüberstellung zur hergebrachten Meinung über Glauben, Zweifel und Kirche zunächst eine Lösung präsentiert, die diese Fragen und Probleme aufgreifen möchte, wird dieser Weg mit der Verpflichtung jedes Einzelnen begründet. Damit erhebt sich die Gemeinschaft moralisch, weil sie eine Schuldigkeit unterstellt, die letztlich in der Gemeinschaft gelöst werden kann. Sie stellt jedem einzelnen die Frage, ob das, was er oder sie glaubt, richtig sei und zeichnet sich zugleich als den Ort und die Gemeinschaft aus,

15 Timecode [0:42-1:36]. 
in der diese Fragen aufgegriffen, behandelt und wohlweislich auch gelöst werden können. Die Darlegung der Opposition zum zuvor konstruierten Problem markiert einen Wendepunkt: Das Allgemein-Abstrakte wird nun konkret spezifisch, aus Zweifel im Leben werden Fragen im Glauben. Und der Ort ist hier: Hier und Jetzt. Die Gemeinschaft präsentiert sich - verkörpert im Individuum, sprachlich einbindend im Wir - als Gegenpol zum Herkömmlichen und Selbstverständlichen und stellt sich gegen eine landläufig vorherrschende Meinung des Ausschlusses von Fragen und Zweifeln in der Kirche. Dabei zieht sich das gleiche Muster durch die Kommunikation: die Trivialisierung des Problems in ihrer Selbstverständlichkeit. Der Weg führt aus der Welt in die Gemeinde, die sich abschließend offenbart:

Als [Name] möchten wir eine Kirche für [Stadt] (-) und $\uparrow$ für [Region] sein, und zwar gerade=auch für diejenigen, die bisher mit dem christlichen Glauben wenig anfangen konnten. Zweifler (-) Skeptiker (-) Suchende=und=Fragende (-) sind uns herzlich willkommen. ${ }^{16}$

Sprachlich wird die lokale Einzigartigkeit hervorgehoben und betont, weil hier der Ort der Auseinandersetzung ist. Zieht man das Visuelle mit ein, werden das Lokale und Einzigartige verdoppelt, der Marktplatz als Erkennungsmarker der Angesprochenen, die sich lokal verorten können. Der geschilderte Weg führt vom universellen Zweifel in die Gemeinde. Das Publikum und die Adressaten sind jedermann. Sie zeigen sich als Merkmalsträger: als Zweifler, Skeptiker, Suchende und Frage, die jede und jeder mehr oder weniger darstellt in der Auseinandersetzung mit der Welt und dem Leben. Es bleibt ein Problem des Menschen, der in der modernen Gesellschaft immerzu ein Suchender ist (vgl. Berger 2015, S. 65f.), weil er wählen und eine Entscheidung treffen muss. Die abschließende Adressierung umreißt den Adressatenkreis, der einerseits lokal begrenzt, andererseits aber wesentlich allgemein jede und jeden betreffen kann.

Ziehen wir den sprachlichen Ausdruck also zusammen, vollzieht sich ein linearer Weg aus der Welt in die Gemeinde. Er wird zur Hinführung: Aus einem universellallgemeinen Problem wird ein individuell adressierbares, und die Lösung findet sich an einem Ort. In der Welt, umgeben von Zweifeln, weil der gesellschaftliche Nomos ungesichert bleibt, bietet die Religion als ,,realissimum“ in besonderer Weise Gewissheit (vgl. Berger 1973, S. 29ff.) und damit die Lösung von Alltagsproblemen sowie kritischer Lebenssituationen. Doch gilt dies nicht mehr? Ist die Kirche nicht in der Lage, diese aufzugreifen? ${ }^{17}$

Aus dem sprachlichen Ausdruck lassen sich drei Elemente hervorheben: der Weg aus der Welt in die Gemeinde als Lösung eines Problems, die für die Gegenüberstellung von Zweifel und Glauben sowie Kirche und Fragen einen Ort der Auseinandersetzung bieten möchte; das zunächst universell-menschliche Problem des Zweifels,

16 Timecode [1:36-1:55].

17 Der Ausschluss von Glaube und Zweifel stützt sich im Christentum auf eine lange Tradition, wenngleich dieselbe Geschichte doch von wiederkehrenden Momenten geprägt ist, in denen Zweifel explizit in Erscheinung treten und theologisch behandelt werden. Schließlich finden sich bereits in der Heiligen Schrift Zeugnisse wie dem ungläubigen Thomas, Jesus am Kreuz oder in privaten Aufzeichnungen Teresas von Kalkutta (vgl. Hoffmann 2018). Zur Unwahrscheinlichkeit von Glaubens- und Wahrheitsgewissheit vgl. auch Soeffner (2013). 
der als Selbstverständlichkeit gedeutet wird und in seiner religiösen Färbung auch hier auf gleichem Wege zur Normalität erhoben wird; und schließlich sind es die angesprochenen Merkmalsträger, die mit der Religion wenig - wohl aber nicht gar nichts - anfangen können, aber durchaus nach einem Ort Ausschau halten, der hier bereitet sein soll.

Was sprachlich als linearer Weg beschreibbar ist, wird körperlich in sich wiederholenden Bewegungen zunächst vertieft: die ideale Ausgangsfigur eines zentral aufrecht gerichteten Sprechers, der mit seinen öffnenden und schließenden Bewegungen der Hände das Gesagte betonend unterstützt und präsentiert. Zugleich zeichnet sich der körperliche Ausdruck durch eine im Verlauf zunehmende Anstrengung und Erschöpfung aus. Der Blick zum Publikum geht verloren, von der idealen Form wird durch Schwingen nach rechts und links, vor und zurück, dem Pendeln um die Mittelachse oder das Herausfallen der Hände aus dem unteren Bildrand abgewichen, um am Ende jedoch wieder in die festumrissene, aufrecht-gerade Ausgangsgestalt zurückzukehren und die Lösung darzubieten. So schwankt der Körper und wird selbst zum Problem des Zweifels. Er fällt aber nicht. Vielmehr zeigt sich der religiöse Experte als die Lösung repräsentierende Gestalt: die aufrecht-angespannte Haltung, geradlinig, zentral, wie das Kreuz als allgemein religiöses Symbol des Christentums.

\subsection{Professionelle Laienhaftigkeit als Inszenierungsmoment}

Welche zentralen Elemente lassen sich nach der skizzenhaften Darstellung herausstellen? Das mediale Arrangement zeichnet sich durch eine schlichte und einfache Gestaltung aus. Die Kamera ist fixiert auf einen Bildausschnitt. Das Geschehen erfolgt lediglich darin. Visuell verortet sich die Kommunikation auf einem Marktplatz und ist zugleich religiös gerahmt. Weltlich-säkularer Raum und religiöse Markierung treten gemeinsam auf. Körperlich und sprachlich vollzieht sich eine Hinführung und Einladung zur Ein- und Umkehr in der Verknüpfung von weltlichem Zweifel und religiöser Vergemeinschaftung.

An dieser Stelle soll der Frage nachgegangen werden, welche kommunikative Gattungen, das heißt, welche vertrauten Formen der Kommunikation leitend für das vorliegende Datum sein können. ${ }^{18}$ Außerdem gilt es, das inhaltlich sich vollziehende Spannungsfeld eines weltlich-säkularen Raums und der religiös markierten Kommunikation näher zu beleuchten.

Zunächst lassen sich Anzeichen vertrauter Formen (massen-)medialer Kommunikation als kommunikative Gattungen beschreiben, wie sie in Formen des Werbefilms, des Imagefilms und dem Wort zum Sonntag auftauchen. So zeichnen die Sprechakte ihrer Struktur nach einen Weg von einem universell-allgemeinen Problem zur religiös spezifischen Lösung. Diesen Weg beschreibt auch Ruth Ayaß (1998) in ihrer Diskussion des Worts zum Sonntag als kommunikativer Gattung. Dieses lasse sich typisch in seinem Aufbau beschreiben und starte meist mit einem areligiösen Beginn als unverfänglichem Einstieg, um die Welt zu adressieren. Darauf folget die Zeichnung eines Jammertals, von Klage und Problem, womit ein religiös-theologisches Problem im Diesseits konstruiert wird, dessen Lösung als Weg ins Licht sogleich

18 Zur kurzen Einführung der kommunikativen Gattungen vgl. Luckmann (2007). 
mitgeliefert wird, um im Schlussakkord eine Wendung in die (göttliche) Zukunft aufzuzeigen (vgl. Ayaß 1998, S. 422ff.). Wie das Wort zum Sonntag verzichtet das Video auf mediale Präsentationsformen und spiegelt eine merkwürdige Verknüpfung von Predigt und Nicht-Predigt gerade auf der sprachlichen Ebene wider.

Das zum Schluss zentrierte, auf die Gemeinde verweisende Symbol erinnert dagegen an die Explikation eines Produkts oder einer Marke, die sprachlich zuvor entfaltet wird. Mit der Fokussierung des Gemeindesymbols lassen sich daher auch Bezüge zum Werbespot herstellen, wodurch die Gemeinde in ihrer Einzigartigkeit - ihrer Opposition - hervorgehoben wird. Als kommunikativer Kern der modernen Alltagskultur ist der Werbefilm allgegenwärtig, vertraut und in unterschiedlichen Variationen vorzufinden. Knoblauch und Raab etwa differenzieren nach Produkt-, Präsentator-, Alltags-, Lebensstil- oder Kunstfilmspot (vgl. Knoblauch und Raab 2002, S. 145ff.). Als wesentliche Merkmale geben sie die Selbstdarstellung des Kommunikators, die Form der Produktdarstellung und schließlich das „Rezipientendesign sowie der soziale und mediale Kontext der Werbung“ an (Knoblauch und Raab 2002, S. 152). Entscheidend sei die „Wahl und die ästhetische Ausgestaltung einer Werbeform [...] stets in Abhängigkeit zum beworbenen Produkt und zur umworbenen Zielgruppe zu sehen" (Knoblauch und Raab 2002, S. 151). Am ehesten ließe sich also von einem Präsentator-Spot ausgehen, in dem jedoch das Problem erst langatmig skizziert werden muss, bis die Lösung und vor allem die von anderen zu unterscheidende Sonderstellung sichtbar wird.

Eine dritte Möglichkeit stellt der Imagefilm dar. Diesen verstehen Bernt Schnettler und Stefan Bauernschmidt als ,Abkömmling des Produkt-, Industrie-, Unternehmens- oder auch Gebrauchsfilms“ (Schnettler und Bauernschmidt 2018, S. 199), als einen kurzen werbenden Film, der Unternehmen, Produkte, Institutionen oder eine Marke porträtiert. In ihrem Aufsatz über universitäre Imagevideos (Bauernschmidt und Schnettler 2018) betonen sie nicht nur die Verflechtung unterschiedlicher Sphären - etwa der Wirtschaft und der Öffentlichkeit -, sie deuten ihn zugleich als Anzeichen zur Bewältigung von Wettbewerb und Krise. Damit lasse er keine eindeutige Grenzziehung zu, sondern werde zum wesentlichen Ausdruck der Grenzüberschreitung. Daher changieren die oftmals nur wenige Minuten dauernden Clips ,einerseits zwischen Werbung und PR und andererseits zwischen (reiner) Beschreibung und (persuasiver) Beeinflussung“ (Schnettler und Bauernschmidt 2018, S. 199). Während Schnettler und Bauernschmidt mediale Möglichkeiten wie Kamerafahrten oder perspektivisch ungewöhnliche Blickwinkel, die sich mit Szenen typischer Aktivitäten abwechseln (vgl. Schnettler und Bauernschmidt 2018, S. 200), hervorheben, verzichtet der vorliegende Fall auf jene medialen Möglichkeiten. Es bleibt eine visuell das Lokale einbindende und religiös markierte Kommunikation, die wesentlich sprachlich ihr Anliegen offenbart.

Die Darlegung des Falls lässt Anleihen an jene drei Gattungen erkennen, die aus massenmedialen Zusammenhängen vertraut sind. Die bewährten Muster zur Lösung kommunikativer Probleme, wie Luckmann Gattungen allgemein beschreibt (vgl. Luckmann 2007, S. 287), werden einerseits kopiert, andererseits aber auch mit ihrer Form gespielt und gebrochen. Schließlich wird ein Produkt in den Vordergrund gestellt, das aber wesentlich erst im Verlauf beschrieben werden muss und 
vielmehr ein Image ${ }^{19}$ herzustellen sucht - , in der Betonung des Unterschieds $\mathrm{zu}$ anderen religiösen Sinnanbietern. Gleichwohl zeigt sich eine stringente Erzählung eines menschlichen Problems: die Erfahrung der Brüchigkeit der Welt im Zweifel, der Konflikt zwischen Glauben und Zweifel und schließlich das Versprechen einer Lösung.

Wie beim Wort zum Sonntag wird ein (religiöses) Problem konstruiert und seine Lösung gleichsam mittransportiert. Die Brüchigkeit der Welt wird durch die Markierung des Problems sichtbar und das Heilsmittel angeboten. Daher ließe sich mit Ayaß betonen, dass hier Religion gerade ,nicht ins Spiel [kommt], wo Menschen zuvor die Brüchigkeit ihrer Lebenswelt erfahren haben“, sondern umgekehrt: „Zunächst wird die Brüchigkeit der Wirklichkeit aufgezeigt, um daraufhin als Heilmittel das Christentum ins Spiel zu bringen“ (Ayaß 1998, S. 444).

Die Aufnahme vertrauter Formen einer massenmedialen Kommunikation verweisen auf eine grundlegende Orientierung an einem professionellen Arrangement. Dagegen markiert der körperliche Ausdruck durch seine Abweichungen in den Bewegungen zugleich Züge des Laienhaften. Ähnliches gilt für die Ausrichtung der Kamera, die Belichtung und den Ton: Die Wahl der Szenerie, ihr Aufbau wie auch die technische Umsetzung folgt professionellen Mustern; dagegen präsentieren das blendende Licht und die Anstrengung sichtbare Brüche. Dem immer wiederkehrenden Abweichen von der Form folgt jedoch stets die Korrektur und Rückbesinnung zur Ausgangsgestalt, weshalb der laienhafte Charakter ebenso professionelle Züge trägt und die Spannung stärker zwischen einer laienhaften Professionalität und professionellen Laienhaftigkeit besteht. Es ist eben kein professionelles, massenmediales Produkt, sondern eine Eigenkreation. Die Orientierung an den Mustern, ihre Kopie und doch eigenständige Bearbeitung, liegt in der Wahl des Mediums begründet, der Gestaltung digital-medialer Kommunikation, die mit relativ geringem Aufwand massenmediale Aufmerksamkeit erreichen kann, wozu es manchmal nur eine Kamera und einen Internetanschluss braucht.

\subsection{Der gläubige Zweifler als Inszenierungstypus}

Abschließend soll der Inszenierungstypus als höchste Verdichtung des kommunikativen Handlungsproblems aufgezeigt werden. Damit rückt auch der Akteur als symbolische Figur nochmals in den Fokus.

Erschüttert das Wort zum Sonntag die natürliche Einstellung zur Welt, indem es existentielle Fragen und Erfahrungen als Probleme markiert, die sinnentleerte Alltagswirklichkeit als Problem der Welt aufruft und die Lösung in der Religion als Moment des Entkommens aus der Ödnis anbietet (vgl. Ayaß 1998, S. 442ff.), grenzt sich das vorliegende Video nicht nur von der Welt ab, sondern zugleich von anderen sinnstiftenden Angeboten. Damit deutet sich eine Auseinandersetzung mit dem an, was Berger mit der These der zwei Pluralismen diskutiert (vgl. Berger 2015, 2017):

\footnotetext{
19 Kurz: „Gesamtheit der im Bewusstsein vorhandener Bilder [...], die ein Akteur oder eine Gruppe von sich selbst, von anderen Individuen, Gruppen, Organisationen, Schichten oder von bestimmten Gegenständen oder materiellen und sozialen Gegebenheiten hat" (Brachfeld 1976 zit. n. Bauernschmidt und Schnettler 2018, S. 170).
} 
die Konkurrenz der Religion zur säkularen Welt, etwa den philosophischen Deutungsmustern, ebenso wie zu anderen Glaubensangeboten. Neben dem Dualismus von Welt und Religion - wie der weltlich-säkulare Raum und religiös-pastorale Text widerspiegeln - wird ein Markt eines religiösen Angebots beschrieben, auf dem der ausgebreitete Widerspruch von Zweifel und Glauben zur Synthese vollzogen werden kann. ${ }^{20}$

Folgt man Bergers These der zwei Pluralismen, verdeutlicht sie die gleichzeitige Auseinandersetzung mit der säkularen Welt und den darin verbreiteten philosophisch-erkenntnistheoretischen Haltungen sowie mit der Konkurrenz zu anderen Anbietern religiöser Deutungsmuster. Diese Auseinandersetzung wird über die Thematisierung des Zweifels gesucht, der als problematisch in Bezug auf den Glauben und Kirche skizziert wird. Wie oben gesehen spielen der zweifelnde Jesus, der ungläubige Thomas oder Teresa von Kalkutta hier keine Rolle. Und wie verhält es sich mit dem durch die Auseinandersetzung von Glaube mit der Welt immerzu bestehenden Zweifel, den Peter Berger und Anton Zijderveld gar als Grundstein eines überzeugenden Glaubens verstehen (vgl. Berger und Zijderveld 2009)?

Die Rolle des Protagonisten lässt sich als Sinnfigur beschreiben, die an das oben skizzierte Spannungsfeld anschließt, weil er gleichermaßen als präsentierender $E x$ perte und präsentierter Laie erscheint. Unterscheidet Max Weber idealtypisch zwischen Priestern, deren Merkmal er als ,Eingestelltheit eines gesonderten Personenkreises auf den regelmäßigen, an bestimmte Normen, Orte und Zeiten gebundenen und auf bestimmte Verbände bezogenen Kultusbetrieb als wesentliches Merkmal“* festhält (Weber 2019, S. 159), und Laien, wird mit dieser Unterscheidung gebrochen. Deutliche Grenzen zwischen religiösen Experten oder Virtuosen und Laien lassen sich hier nicht mehr trennscharf bilden. Vielmehr tritt der Experte für den Zweifel selbst als Zweifelnder in Erscheinung, der schwankt, bemüht ist, die Form zu wahren, sich wie das gesamte Kommunikat im Wechselspiel der Spannungen befindet, als wäre die Entscheidung für eine Seite gar nicht möglich.

Mit Plessner lässt es sich vielleicht gerade so erklären: weil „,der Mensch auch praktisch nach zwei Seiten [strebt], in die Unbewußtheit, Ursprünglichkeit, Naivität und in die Bewußtheit, in das Raffinement der Überlegung, der Selbstbeobachtung und Selbstbeherrschung“ (Plessner 2019, S. 66), vielleicht oder gerade wegen der Zweideutigkeit alles Psychischen, als trage ihr Hervorwagen das ,Risiko der Lächerlichkeit“ (Plessner 2019, S. 70) in sich. Schließlich - so Plessner - ist das ,Streben nach Geltung, nach praktischer Macht, nach Illusion zu verneinen und radikal ernst zu machen mit der Umkehr zur Preisgabe der irdischen Güter und Werte" (Plessner 2019, S. 77). So bietet die Gemeinschaft zwar Geborgenheit, Religion Stabilität und Gewissheit, doch das moderne Individuum steht in diesem Zwischenspiel. Erst der wahrhaft Gläubige ,nimmt das Risiko der Niedrigkeit und Lächerlichkeit auf sich, mit heiterer Seele, denn er weiß, daß ihm Gott dieses Risiko abgenommen hat wie allen, auch wenn sie es nicht wahrhaben wollen, bis ans Ende der Tage“ (ebd.). Und dieser Schritt - so ist zu vermuten - steht hier noch aus. Denn es vermag sich symbolisch das Dazwischen zu etablieren, der Akteur als symbolische Figur, weil Symbole an der „Grenze der Kommunikation“ arbeiten, in der Vermittlung der Grenzen der

20 Zur Übersicht des Marktmodells der Religion vgl. Pickel (2011, S. 198ff). 
alltäglichen Kommunikation (vgl. Soeffner 2008, S. 63). Sie sind eben ,nicht nur Statthalter des Außeralltäglichen im Alltag, sondern sie fungieren zugleich auch als unübersehbarer Hinweis auf die permanent drohende Gefahr des Zusammenbruchs des Mundanen, des Versagens der alltäglichen Ordnungen und damit auf die Stabilität der menschlichen Existenz“ (ebd.). Schließlich fassen sie Spannung, Konkurrenz und Widerspruch zusammen, wie das christliche Kreuz Tod und Auferstehung gleichermaßen bedeutet (vgl. Soeffner 2008, S. 60f.). Der Protagonist wird zum Symbol des zweifelnden Gläubigen, zum Zusammenzug von Harmonie und Widerspruch, in dem beide Seiten oszillieren, wie sie das Kommunikat durchziehen.

Der medialen Ausgestaltung ist daher auf der einen Seite die Spannung zwischen laienhafter Professionalität und professioneller Laienhaftigkeit inhärent, die sich als Anbindung an und Abweichung von vertrauten Formen (massen-)medialer Kommunikation medial wie körperlich zeigt, durchaus eine Konsequenz der eigenen medialen Gestaltbarkeit der Kommunikation im digital-medialen Zusammenhang. Auf der anderen Seite durchzieht das Kommunikat die Spannung zwischen Religion und weltlich-säkularer Sphäre, die als Bühne gebraucht wird. Auf ihr tritt der Protagonist in der Sinnfigur des zweifelnden Gläubigen und gläubigen Zweiflers auf. Das jedoch lässt ihn - und uns - in einem Dazwischen zurück, als stünde der notwendige Sprung noch bevor.

Das vermag abschließend das eigentliche Inszenierungsmoment zu sein, das Oszillieren zwischen den Polen, dargestellt im Spannungsfeld von Professionalität und Laienhaftigkeit, Religiosität und Säkularität, Glaube und Zweifel, das sich medial ausbreitet und das der Protagonist in der Sinnfigur körperlich mitträgt, wenn er uns als gläubiger Zweifler den Weg weist. Visuell ist es die Verortung in der Einzigartigkeit und zugleich in einer allgemein symbolisch eingeholten weltlich-säkularen Öffentlichkeit auf einem Markt der Möglichkeiten, die Religion im säkularen Raum auftreten lässt. Es ist der körperliche Ausdruck, der wankt, aber dennoch fest verankert im sprachlichen Ausdruck nur eine Richtung kennt. Das als Gegensatz und eigentliches Ausschlusskriterium von Kirche konstruierte Problem wird zum eigentlichen Bezugspunkt: der Einbezug von Zweifeln (und Fragen) am Glauben in der Kirche. Mediales Arrangement, räumliches Setting sowie körperliche und sprachliche Ausdrucksmittel verdeutlichen zusammengenommen jene Strukturelemente als Spannung zwischen professioneller Laienhaftigkeit und laienhafter Professionalität durch Vorbereitung, Orientierung und Ausführung, der im Bildausschnitt visuell symbolisierten Religion im weltlich-säkularen Raum bei gleichzeitiger visueller wie sprachlicher Dominanz der spezifisch einzigartigen Lokalität, weshalb der Weg nicht aus der Welt in die Kirche, sondern explizit in diese Gemeinde führt.

\section{Konservative Popularität: abschließende Bemerkungen}

Der Beitrag untersuchte mithilfe der Wissenssoziologischen Videohermeneutik einen exemplarischen Fall religiöser Kommunikation einer Freikirche. Dies geschah verkürzt und skizzenhaft. Die dargelegten Ergebnisse sind also zunächst fallspezifisch und besonders. Die Grundlagen der Methode liegen in der sozialwissenschaftlichen Hermeneutik, wie sie von Hans-Georg Soeffner entwickelt wurde, die neben ihrer 
fallspezifischen Orientierung zugleich idealtypisch angelegt ist (vgl. Soeffner 2004, S. 118f.). Es ist die Fallspezifik, die dadurch zum Vorschein kommt, beziehungsweise ,die Besonderheit des Falles im Rahmen der für ihn objektiv möglichen Kontexte und Sinnwelten" (Soeffner 2004, S. 138), weil das Ziel immer heißen muss, das Allgemeine im Individuellen sichtbar zu machen (vgl. Soeffner 2004, S. 154f.). Die Analyse ist damit immer exemplarisch, weil ,per se Fallanalyse“ (Soeffner 2004, S. 158). Hierüber aber trägt sie die Möglichkeit der Entwicklung und Erklärung eines (Ideal-)Typus (vgl. ebd.). Der Fallvergleich steht aus, aber die aufgezeigten Merkmale deuten bereits auf einen umfassenderen Zusammenhang. Religion beziehungsweise religiöse Akteure und Gemeinden stehen vor der Herausforderung, mit einer Welt umzugehen, in der sie Sinnanbieter neben anderen sind. Die Verwendung medialer Verbreitungsmöglichkeiten ist dabei nichts Neues. Wie Oliver Krüger festhält, ,,berühren [Medien] die Fundamente der Kultur, der Religion und der gesellschaftlichen Ordnung, da sie epistemologische Fragen nach der Gültigkeit und Wahrheit der sinnlichen Erfahrung, des Gehörten und Gesehenen aufwerfen“" (Krüger 2012, S. 77). Medien stehen stets in der Spannung zwischen der Vermittlungsmöglichkeit und dem Problem der Vermittlung, sowohl Dinge erfahrbar zu machen als auch Zweifel zu säen, ,ob diese vermittelten Erfahrungen echt und authentisch sind“ (ebd.), weshalb sie einerseits als Verkündigungsinstrument auch in den Religionen dienen, andererseits aber durchaus als Bedrohung gesehen werden können (vgl. Krüger 2012, S. 32). Religionsgeschichtlich zeigen sich die Einbindung neuer Medien in die Religion oder eine „Medienreligion“ als „Normalfall“, stehen „Religionsgeschichte und Mediengeschichte schließlich im Wechselspiel“, weil die Religion Medien zur Aufmerksamkeitssteigerung aufgreift (Krüger 2012, S. 40).

Der dargelegte Fall verdeutlicht eine Möglichkeit religiöser Kommunikation in der Einladung beziehungsweise Verbreitung und Anwerbung. In seiner Ausgestaltung greift er nicht nur vertraute Formen kommunikativer Gattungen auf, sondern variiert sie und nutzt ihre grundlegenden Muster zur Verbreitung der eigenen Botschaft. Dadurch lässt sich die im Material explizierte und unaufgelöste Spannung von laienhafter Professionalität und professioneller Laienhaftigkeit erkennen, die aus dem permanenten Wechselspiel einer aus massenmedialen Zusammenhängen vertrauten Form und der spezifischen Gestaltung und Variation herrührt. Was sich als Merkmal der medialen Gestaltung zeigt, eine Positionierung im Dazwischen, eben sowohl vertraute Form als auch ihr Bruch zu sein, vollendet sich schließlich in der Darstellung des Protagonisten, der als religiöser Experte auftritt und zugleich in seinen Bewegungen vor der Kamera den Charakter des gerade nicht selbstverständlichen Darstellers hervorhebt. Es sind die Abweichungen des Körpers, sein Pendeln und Schwanken, die dem geradlinigen Weg aus der Welt in die Gemeinde, vom Zweifel zum Glauben entgegenstehen. In ihm fallen das Handlungsproblem und dessen Lösung zusammen. Er wird zur symbolischen Figur, die Frage und Antwort gleichermaßen vorführt, gerade entgegen der eigentlichen Konsumwerbung, die zwischen Problem und Lösung trennen muss (Knoblauch und Raab 2002).

Offen bleibt, wie Religion auf die Welt tatsächlich reagiert. Mit Berger ließe sich an dieser Stelle argumentieren, dass das Problem entweder fundamentalistisch oder relativistisch zu lösen sei, beide Möglichkeiten jedoch Folgeprobleme wie fortwährende Konflikte, totalitärer Zwang oder Unterminierung des moralischen 
Konsenses aufweisen können (vgl. Berger 2015, S. 33f.; auch S. 98f.), weshalb er vermutet, dass die Lösung irgendwo dazwischen liegt. Dass aber der Zweifel ins Zentrum gerückt wird, kann als Hinweis auf die Auseinandersetzung mit der pluralistischen Welt gesehen werden, ist er doch „die Achse, um die die Dynamiken des Pluralismus wirbeln“ (Berger 2015, S. 99), weil der Zweifel im Denken „den Glauben beinahe immer - vielleicht nicht bei den ,religiösen Virtuosen“ nach Weber, aber bei der großen Mehrheit der gewöhnlichen Gläubigen“ (ebd.) mitbestimmt. Dies wird in der körperlichen Sinnfigur visuell sichtbar, weshalb sie als zweifelnder Gläubiger zu beschreiben ist und auf jenen Typus zielt, der - an anderer Stelle im Sinne der Grounded Theory (vgl. Glaser und Strauss 1998; Strauss 2004) - in kontrastierenden Fallvergleichen weiter zu konkretisieren ist.

Das Inszenierungsmoment in der starken Anknüpfung an vertraute Gattungen verweist an dieser Stelle zunächst auf einen konservativen medialen Stil, der dem Zweifelnden Stabilität, Sicherheit und Orientierung liefert. Die Verwendung dieser Formen in der Verbreitung der eigenen Botschaft ist jedoch ebenso Zeugnis der Aufnahme populärer Kommunikationsformen beziehungsweise populärkultureller Elemente (vgl. Knoblauch 1999; Schnettler 2014). Die Möglichkeiten digitalmedialer Kommunikation gerade in ihrer Gestaltbarkeit lassen das zu, sie öffnen aber auch das Feld, diese ganz anders auszurichten, je nachdem, welches Publikum es zu erreichen gilt.

\section{Anhang}

Tab. 1 Transkriptionskonvention

\begin{tabular}{ll}
\hline$(-)(--)(--)$ & Sehr kurze bis kurze Pause (unter einer Sek.) \\
$;$ [bzw.] . & Schwach bzw. stark fallende Intonation \\
, [bzw.] ? & Schwach bzw. stark steigende Intonation \\
Betont & Betont gesprochen \\
$\mathrm{Na}-$ & Abgebrochenes Wort \\
$=$ mal & Schneller Anschluss \\
$\downarrow$ & Fallende Intonation in einer Silbe \\
$\uparrow$ & Steigende Intonation in einer Silbe \\
\hline
\end{tabular}


Funding Open Access funding enabled and organized by Projekt DEAL.

Open Access Dieser Artikel wird unter der Creative Commons Namensnennung 4.0 International Lizenz veröffentlicht, welche die Nutzung, Vervielfältigung, Bearbeitung, Verbreitung und Wiedergabe in jeglichem Medium und Format erlaubt, sofern Sie den/die ursprünglichen Autor(en) und die Quelle ordnungsgemäß nennen, einen Link zur Creative Commons Lizenz beifügen und angeben, ob Änderungen vorgenommen wurden.

Die in diesem Artikel enthaltenen Bilder und sonstiges Drittmaterial unterliegen ebenfalls der genannten Creative Commons Lizenz, sofern sich aus der Abbildungslegende nichts anderes ergibt. Sofern das betreffende Material nicht unter der genannten Creative Commons Lizenz steht und die betreffende Handlung nicht nach gesetzlichen Vorschriften erlaubt ist, ist für die oben aufgeführten Weiterverwendungen des Materials die Einwilligung des jeweiligen Rechteinhabers einzuholen.

Weitere Details zur Lizenz entnehmen Sie bitte der Lizenzinformation auf http://creativecommons.org/ licenses/by/4.0/deed.de.

\section{Literatur}

Ayaß, Ruth. 1998. Wort zum Sonntag als kommunikative Gattung. In Religion als Kommunikation, Hrsg. H. Tyrell, V. Krech, und H. Knoblauch, 417-445. Würzburg: Ergon-Verlag.

Bauernschmidt, Stefan, und Bernt Schnettler. 2018. Das Rätsel hochschulischer Imagefilme: Eröffnungssequenzen. Einstieg in die Analyse visueller Kommunikationsgattungen. In Knowledge in Action. Neue Formen der Kommunikation in der Wissensgesellschaft, Hrsg. E. Lettkemann, R. Wilke, und H. Knoblauch, 165-187. Wiesbaden: Springer.

Berger, Peter L. 1973. Zur Dialektik von Religion und Gesellschaft. Elemente einer soziologischen Theorie. Frankfurt a.M.: Fischer. Übers. v. Monika Plessner.

Berger, Peter L. 1980. Der Zwang der Häresie. Religion in der pluralistischen Gesellschaft. Frankfurt a.M.: Fischer. Aus dem Amerikanischen v. Willi Köhler.

Berger, Peter L. 2015. Altäre der Moderne. Religion in pluralistischen Gesellschaften. Frankfurt a.M., New York: Campus.

Berger, Peter L. 2017. Zwei Pluralismen. In Zwei Pluralismen. Positionen aus Sozialwissenschaft und Theologie zu religiöser Vielfalt und Säkularität, Hrsg. P.L. Berger, S. Steets, und W. Weiße, 17-27. Münster, New York: Waxmann.

Berger, Peter L., und Thomas Luckmann. 2020. Religionssoziologie als Wissenssoziologie. In Religiöse Kommunikation und weltanschauliches Wissen. Wissen, Kommunikation und Gesellschaft, Hrsg. B. Schnettler, T. Szydlik, und H. Pach, 21-32. Wiesbaden: Springer.

Berger, Peter L., und Anton C. Zijderveld. 2009. Lob des Zweifels. Was ein überzeugender Glaube braucht. Freiburg i.B.: Kreuz.

Bourdieu, Pierre. 2000. Das religiöse Feld. Texte zur Ökonomie des Heilsgeschehens. Konstanz: UVK.

Glaser, Barney G., und Anselm L. Strauss. 1998. Grounded Theory. Strategien qualitativer Forschung. Bern: Huber.

Herbrik, Regine. 2013. „Du sollst dir kein Bildnis machen“-Zur Verwendung von Video- und Bildmaterial und ihrer Bedeutung für die emotionalen Stile christlicher Gemeinden heute. In Visuelles Wissen und Bilder des Sozialen. Aktuelle Entwicklungen in der Soziologie des Visuellen, Hrsg. P. Lucht, L.M. Schmidt, und R. Tuma, 141-156. Wiesbaden: Springer.

Hoffmann, Veronika. 2018. zweifeln und glauben. Stuttgart: camino.

Knoblauch, Hubert. 1999. Populäre Religion. Markt, Medien und die Popularisierung der Religion. In Diesseitsreligion. Zur Deutung der Bedeutung moderner Kultur, Hrsg. A. Honer, R. Kurt, und J. Reichertz. Konstanz: UVK.

Knoblauch, Hubert. 2001. Ekstatische Kultur. Zur Kulturbedeutung der unsichtbaren Religion. In Moderne Zeiten. Reflexionen zur Multioptionsgesellschaft, Hrsg. A. Brosziewski, T.S. Eberle, und C. Maeder, 153-167. Konstanz: UVK.

Knoblauch, Hubert. 2006. Religion und Soziologie. In Religion in der modernen Lebenswelt. Erscheinungsformen und Reflexionsperspektiven, Hrsg. B. Weyel, W. Gräb, 277-295. Göttingen: Vandenhoeck \& Ruprecht. 
Knoblauch, Hubert. 2017. Die kommunikative Konstruktion der Transzendenz und die populäre Religion. In Religion soziologisch denken, Hrsg. H. Winkel, K. Sammet, 221-241. Wiesbaden: Springer.

Knoblauch, Hubert, und Jürgen Raab. 2002. Der Werbespot als kommunikative Gattung. In Die Werbung der Gesellschaft, Hrsg. H. Willems, 139-154. Wiesbaden: Westdeutscher Verlag.

Krüger, Oliver. 2012. Die mediale Religion. Probleme und Perspektiven der religionswissenschaftlichen und wissenssoziologischen Medienforschung. Bielefeld: transcript.

Luckmann, Thomas. 2007. Grundformen der gesellschaftlichen Vermittlung des Wissens: Kommunikative Gattungen. In Lebenswelt, Identität und Gesellschaft, 272-293. Konstanz: UVK.

Luckmann, Thomas. 2014. Die unsichtbare Religion. Frankfurt a.M.: Suhrkamp.

Oevermann, Ulrich. 2000. Die Methode der Fallrekonstruktion in der Grundlagenforschung sowie der klinischen und pädagogischen Praxis. In Die Fallrekonstruktion. Sinnverstehen in der sozialwissenschaftlichen Forschung, Hrsg. K. Kraimer, 58-156. Frankfurt a. M.: Suhrkamp.

Pickel, Gert. 2011. Religionssoziologie. Eine Einführung in zentrale Themenbereiche. Wiesbaden: Springer VS.

Plessner, Helmuth. 2019. Der Kampf ums wahre Gesicht. Das Risiko der Lächerlichkeit. In Grenzen der Gemeinschaft. Eine Kritik des sozialen Radikalismus, 58-78. Frankfurt a.M.: Suhrkamp.

Raab, Jürgen. 2008. Visuelle Wissenssoziologie. Theoretische Konzeption und materiale Analysen. Konstanz: UVK.

Raab, Jürgen, und Marija Stanisavljevic. 2018. Wissenssoziologische Videohermeneutik. In Handbuch Qualitative Videoanalyse, Hrsg. C. Moritz, M. Corsten, 57-71. Wiesbaden: Springer.

Reichertz, Johannes. 2002. Zeit zur Aussat? - Werbung für die Kirche zwischen Reklame und Bekenntnis. In Die Gesellschaft der Werbung. Kontexte und Texte. Produktionen und Rezeptionen. Entwicklungen und Perspektiven, Hrsg. H. Willems, 324-341. Wiesbaden: Westdeutscher Verlag.

Schnettler, Bernt. 2014. Transzendenzerfahrung und populäre Religion. In Zwischen Säkularisierung und religiöser Vitalisierung. Religiosität in Deutschland und in Polen im Vergleich, Hrsg. M. Hainz, G. Pickel, D. Pollack, M. Libiszowska-Zoltkowska, und E. Firlit, 271-277. Wiesbaden: Springer.

Schnettler, Bernt, und Stefan Bauernschmidt. 2018. Bilder in Bewegung. Visualisierung in der Wissenschaftskommunikation. In Das Bild als soziologisches Problem. Herausforderungen einer Theorie visueller Sozialkommunikation, Hrsg. M.R. Müller, H.-G. Soeffner, 197-208. Weinheim, Basel: Beltz Juventa.

Soeffner, Hans-Georg. 2004. Hermeneutik. Zur Genese einer wissenschaftlichen Einstellung durch die Praxis der Auslegung. In Auslegung des Alltags - Der Alltag der Auslegung. Zur wissenssoziologischen Konzeption einer sozialwissenschaftlichen Hermeneutik, Hrsg. Hans-Georg Soeffner, 114-159. Konstanz: UVK.

Soeffner, Hans-Georg. 2008. Symbolische Präsenz: unmittelbare Vermittlung - zur Wirkung von Symbolen. In Phänomenologie und Soziologie. Theoretische Positionen, aktuelle Problemfelder und empirische Umsetzungen, Hrsg. J. Raab, M. Pfadenhauer, P. Stegmaier, J. Dreher, und B. Schnettler, 53-64. Wiesbaden: VS.

Soeffner, Hans-Georg. 2013. Religion und Kultur des Individuums. Zwölf Thesen. In Religionshybride. Religion in posttraditionalen Kontexten, Hrsg. P.A. Berger, K. Hock, und P. Klie, 285-304. Wiesbaden: Springer VS.

Strauss, Anselm L. 2004. Methodologische Grundlagen der Grounded Theory. In Methodologie interpretativer Sozialforschung. Klassische Grundlagentexte, Hrsg. J. Strübing, B. Schnettler, 429-451. Konstanz: UVK.

Strübing, Jörg. 2008. Grounded Theory. Zur sozialtheoretischen und epistemologischen Fundierung des Verfahrens der empirisch begründeten Theoriebildung. Wiesbaden: VS.

Traue, Boris, und Anja Schünzel. 2019. YouTube und andere Webvideos. In Handbuch Methoden der empirischen Sozialforschung, Hrsg. N. Baur, J. Blasius, 1065-1077. Wiesbaden: Springer.

Weber, Max. 2019. Wirtschaft und Gesellschaft. Religiöse Gemeinschaften. In Max Weber-Gesamtausgabe, Band I/22,2, Hrsg. H.G. Kippenberg https://doi.org/10.1628/978-3-16-158139-7.

Wernet, Andreas. 2019. Wie kommt man zu einer Fallstrukturhypothese? In Vom Fall zur Theorie. Auf dem Pfad der rekonstruktiven Sozialforschung, Hrsg. D. Funcke, T. Loer, 57-84. Wiesbaden: VS.

Weyer, Johannes. 2017. Die Echtzeitgesellschaft. Wie smarte Technik unser Leben steuert. Frankfurt a.M.: Campus.

Ziemann, Andreas. 2018. Medien und Gesellschaft. In Mediensoziologie. Handbuch für Wissenschaft und Studium, Hrsg. D. v. Hoffman, R. Winter, 57-70. Baden-Baden: Nomos.

Zimmerling, Peter. 2018. Charismatische Bewegungen. Göttingen: Vandenhoeck \& Ruprecht. 